\title{
Effects on community structure of a pollutant gradient - summary
}

\author{
R. M. Warwick* \\ Plymouth Marine Laboratory (West Hoe), Prospect Place, The Hoe, Plymouth PL1 3DH, United Kingdom
}

\section{MATERIAL AND METHODS}

Seven $0.1 \mathrm{~m}^{2}$ replicate Day grab samples for macrofauna were taken at 6 sites $(A, B, C, D, E, G)$ in Frierfjord/ Langesundfjord, Norway, sieved at $1.0 \mathrm{~mm}$ and preserved in formalin. Five $5.6 \mathrm{~cm}$ internal diameter replicate Craib core samples for meiofauna were also taken from 6 sites $(A, B, C, D, E, F)$, sieved at $63 \mu \mathrm{m}$ and preserved in formalin. Sediment samples for microbial analysis were subsamples of one meiofaunal core from each site.

For the mesocosm experiment at Solbergstrand, twenty $0.25 \mathrm{~m}^{2}$ USNEL box core samples of sediment were taken from Bjørnehodet Bay and transferred undisturbed in plastic liners to the mesocosm basins, 5 being allocated at random to each of the 4 treatments $-C$ : control, L: low, M: medium, H: high dosing of a copper and diesel oil mixture. After an exposure period of ca 3 mo, two $9.3 \mathrm{~cm}$ internal diameter cores for macrofauna, four $5.6 \mathrm{~cm}$ cores for meiofauna and one $0.5 \mathrm{ml}$ surface sediment sample for microbes were taken from 4 of the 5 boxes in each basin, and processed as above

All macrofauna were identified to species level, counted and weighed (wet wt). Only 4 of the 7 field replicates were analysed. Field meiofauna were enumerated to major taxon level in 4 of the 5 replicates at each site; copepods were identified to species in 3 of these replicates, nematodes in 2 replicates. Only nematodes and copepods were enumerated (to species level) in the mesocosm experiment, based on subsamples of $16 \%$ of each sediment core, the 4 subsamples from each box being combined. Microbes were only identified to major taxa both in the field and mesocosm samples, and abundance and biomass (from volume measurements) were determined. A benthic biomass size-spectrum was constructed from the microbial, meiofauna and macrofauna data.

\footnotetext{
- This summary was written following discussions amongst all contributors to this section
}

\section{ANALYSIS OF DATA}

Four distinct stages in the identification of pollution effects on benthic communities can be recognised, each with its own set of analytical techniques.

(1) Multivariate methods used to discriminate between sites based on their faunal attributes. Three broad categories of techniques have been applied.

Classification. At the workshop, hierarchical agglomerative clustering based on group-averaging of Bray-Curtis similarity measures was used throughout. TWINSPAN was used for Indicator Species Analysis (field meiofauna only).

Ordination. Several techniques were compared, including Multidimensional Scaling (MDS), Detrended Correspondence Analysis (DECORANA), Principal Components Analysis (PCA) and Reciprocal Averaging (RA).

Discrimination tests. The significance of differences between field sites or mesocosm treatments was tested using Analysis of Similarity (ANOSIM), Roy's Greatest Root Criterion and Malhanobis' Distance tests; the latter were followed by Canonical Discriminant Analysis (CDA).

The effects of various strengths of transformation of the abundance and biomass data on the results of the multivariate analyses were tested.

(2) Univariate methods used to determine levels of disturbance or 'stress' at given sites. These can be divided into 2 categories.

Methods which can only be used in a comparative manner between sites along a spatial or temporal gradient. Those applied were: number of taxa (S), total abundance $(A)$, total biomass $(B), A / S$ (abundance ratio), $B / A$ (size ratio), abundance and biomass group distributions, dominance distributions, diversity and evenness indices $\left(H^{\prime}, D, J\right.$, Hill's diversity numbers), comparison of functional (e.g. trophic) groups, biomass spectra.

$0171-8630 / 88 / 0046 / 0207 / \$ 03.00$ 
Methods which can be applied to single sites without the need for reference samples. Those applied were: identification of indicator organisms (species or higher taxa), abundance/biomass comparison curves (ABC).

(3) Methods of correlating (1) and (2) above with pollution levels. Care must be taken to identify such natural confounding variables as depth, sediment type physical disturbance etc, when relating the observed differences in faunal attributes between sites to chemical measurements of pollution levels, or proximity to pollution sources. The method used for multivariate analyses (ordinations) was to superimpose levels of pollutants and some of the more obvious natural variables onto the site configurations to provide visual correlations. The univariate indices have been related to pollutant levels using standard statistical techniques (principally for the microbial studies).

(4) Methods to establish whether pollution causes the observed pattern of site differences. The level of community reponse to measured levels of pollutants in controlled experiments was studied in an attempt to provide this test, which at the workshop constituted the mesocosm experiment. Methods of analysing these data were essentially the same as those given in (1) and (2) above.

\section{RESULTS}

\section{Field macrofauna}

(1) The different multivariate analyses using a variety of transformations were in general agreement with each other, although there were some differences in detail. Three major groups of sites were seen: $B+C+D$, $E+G$ and $A$. Some analyses separated the replicates at $D$ from those of $B+C$ ( $B$ and $C$ were never divided) and most analyses separated $E$ from $G$, though the latter were always closer to each other than any other site. To test the idea that species in the intermediate abundance classes were mainly responsible for the observed structure, MDS analyses were performed on the 19 species in these classes. Although these reproduced the structure of the full data set rather closely, they did so no better than any randomly selected subset of 19 species. This indicates strong site differences and a high degree of redundancy in the data. Such redundancy could be exploited by laboratories with limited taxonomic expertise, or in regions of the world where the fauna is poorly documented, if identification to species level were not necessary and sufficient information remained using higher taxonomic groupings. The workshop data for both species abundance and biomass were aggregated at the family and phylum level. At the family level, MDS on the abundance data produced results which were virtually identical to the species level configurations, the same being true for the untransformed biomass data; with the 4 th root transformed biomass data there was an appreciable improvement in the separation of replicates from certain sites. At the phylum level, groupings based on abundance data that were apparent at the species and family level largely broke down, but the biomass data faithfully reproduced these groupings with surprisingly little loss of information.

(2) Comparison of a wide variety of univariate measures between sites indicated that the communities at Sites $B$ and $C$ were the most stressed, followed in decreasing order by $D, E, G$ and $A$. Of the methods which did not require inter-site comparison, indicator taxa suggested that Sites B, C and D were stressed and that Sites $A$ and $G$ were unpolluted, whereas the pollution status at Site $E$ was equivocal. $A B C$ plots indicated that Sites $C$ and $D$ were intermediate between the 'moderately polluted' and 'grossly polluted' conditions, Sites B and E were 'moderately polluted' and Sites A and $G$ 'unpolluted'; aggregation of the data to family level gave exactly the same results.

(3) Two pollution variables (metal and PAH concentrations) and 2 natural variables (water depth and median sediment grain size) were superimposed on the site configurations in the species abundance MDS (under 4 th root transformation). Neither sediment type, metals or PAH correlated closely with the site configuration, whereas water depth did. Furthermore, the sites where the univariate measures indicated the highest degree of stress ( $B, C$ and $D)$ were not those with the highest measured levels of pollutants but were at the greatest water depths. The most parsimonious explanation for differences between communities in the multivariate analyses is therefore that they result from the well-known effect of water depth on benthic community type, and the clear indications of stress derived from the univariate techniques at the deeper sites do not result from inputs of pollutants at the head of the fjord but to some other depth-related character. Seasonal anoxia in the deeper basins of the fjord is welldocumented, and is the most likely cause of stress at these deep sites. For pollution surveys of heterogeneous geographic regions where the differences in nonpollution related ('nuisance') variables between sites are great, it is postulated that multivariate analyses at the higher group (e.g. phylum) level may more closely reflect the pollution gradient. It is well documented that pollution modifies community structure at this higher level, whereas differing environmental conditions (e.g. water depth and sediment granulometry) may affect community structure more by replacement at the species level. 


\section{Field meiofauna}

A major drawback to the use of meiobenthos in programmes for monitoring the effects of pollution has been that the necessary expertise in identifying these organisms to species level is lacking in most laboratories, and in any case a taxonomic literature approaching adequacy is only available in Northern Europe and perhaps North America. An important element of the meiofauna study was therefore to analyse the data assuming that less taxonomic rigour was possible.

(1) As with the macrofauna, the different multivariate analyses gave generally similar results, with some differences in detail. The analyses were very robust to the aggregation of the nematode and copepod species data into higher taxa. The use of nematode feeding groups and the abundances of major taxa also produced similar results. With different numbers of replicates for nematodes, copepods and major taxa it was difficult to compare the resolving power of the different hierarchical groupings of taxa, but generally copepod species were best in separating all sites in at least some analyses, although the nematodes were more robust to aggregation into higher taxa.

(2) Many of the univariate measures listed earlier are not applicable or have not yet been well developed for use with meiofauna; heavy reliance has therefore been placed on diversity measures. For a range of diversity indices, Site $F$ had a significantly lower diversity of nematodes and copepods both for species and higher taxa. For meiofauna groups, this trend was not apparent for all diversity indices. Plotting $k$-dominance curves for nematode species indicated that Site $F$ clearly had the lowest diversity, followed by $E$ and then $B$, and $A, C$ and $D$ had the highest diversity and were indistinguishable from each other. Similar plots for copepods showed that $D$ had the highest diversity, but otherwise there was no clear separation of curves.

(3) The marked separation of Site $F$ in the multivariate analyses, and its low meiofaunal diversity relative to the other sites, coincided with the much higher PAH levels at that site and the much higher levels of certain metals, particularly cadmium. This site was of intermediate water depth, so that its distinctive characteristics did not relate to naturally occurring depth-related environmental variables. Thus there was correlative evidence that the meiofauna were affected by pollution. In many of the multivariate analyses the remaining Sites A to E were grouped in the same way as for the comparable macrofaunal analyses. Therefore, naturally occurring depth-related factors rather than pollution were implicated, although, unlike the macrofauna, the meiofaunal assemblages at the deeper sites $(B, C$ and $D)$ showed no signs of stress.

\section{Field microbes}

(1) No multivariate analyses were performed on the microbial data.

(2) There were significant differences in abundance of bacterial rods + cocci and microflagellates among Sites A to $F$, with the combined inner sites $(D, E, F)$ having significantly lower abundances of bacterial filaments, rods+cocci and microflagellates than the combined Langesundfjord sites $(A, B, C)$. The same trend was apparent in the biomasses of all 3 microbial groups.

(3) Microbial abundance and biomass did not correlate closely with water depth or sediment granulometry. Concentrations of lead and manganese in the sediments were negatively correlated with microflagellate biomass, and zinc concentrations were negatively correlated with bacterial biomass. However, pairwise comparisons showed that the most highly polluted Site F was not significantly different from Sites $D$ and $E$ in the abundances of any of its microbial components, and no causal inferences could be drawn from these correlations.

\section{Field biomass spectra}

(1) For field sites, MDS was used for the macrofaunal size range only, treating the $\times 2$ geometric size classes as 'species' in a normal analysis. Sites B, C and D were grouped together, as were Sites $A$ and $E$, reflecting a similar split to that found for macrofauna and meiofauna taxa.

(2) For the complete size spectrum, there was an overall decrease in biomass of most size classes within the microbial, meiofaunal and macrofaunal categories, from Sites A to E. Spectra had a higher degree of 'bumpiness' at Sites D, E and $F$ than at $A, B$ and $C$, with more pronounced biomass troughs between meiofauna and macrofauna peaks.

(3) The differences between the size spectra were not obviously correlated with pollution levels, and unfortunately no macrofauna samples were available from the most heavily polluted site $(F)$, where any pollutioninduced modifications to the spectrum would have been most apparent. For the macrofaunal component, MDS of the size classes corroborated the equivalent taxonomic analyses, suggesting the same causation. There was a trend to reduced average size of organisms in the macrofaunal assemblages which showed the most stress.

\section{Mesocosm macrofauna}

(1) Most multivariate analyses failed to demonstrate clear differences between treatments. An exception 
was a MANOVA analysis on the first 5 principal components of (log) species abundances, the treatment means being ordered $\mathrm{C}-\mathrm{L}-\mathrm{M}-\mathrm{H}$ on the first canonical variate axis (a 1 in 12 chance of occurring if there were no treatment effects). On balance, evidence for community change with treatment must be regarded as marginal.

(2) Most of the univariate analyses used failed to demonstrate treatment effects, perhaps due to the fact that the small core size failed to sample the large biomass dominants adequately. However, the high dose treatment had an uneven distribution of species abundance groups, which is indicative of moderate organic enrichment. Seven species in the middle abundance groups showed trends of increasing or decreasing abundance with treatment level commensurate with their predicted behaviour based on previous studies of organic enrichment in the field.

(3) Differences in species composition and community structure between mesocosm treatments were minimal, certainly not approaching the differences between the field sites. The main reasons for this may be the short time scale of the experiment relative to the generation time of the macrofaunal organisms, problems of macrofaunal recruitment to the mesocosm boxes, the fact that pollutants did not penetrate the sediments over the timescale of the experiment and the small sample size. For these reasons it was not possible to test in this experiment whether the measured pollutant levels in the field were capable of causing community differences.

\section{Mesocosm meiofauna}

(1) For both nematode and copepod species abundances, multivariate analyses revealed some significant differences between the high dose and other treatments. PCA produced better separation of the $\mathrm{H}$ replicates than other ordinations, the treatment level means on $\mathrm{PC} 2$ again ranking as $\mathrm{C}-\mathrm{L}-\mathrm{M}-\mathrm{H}$. However, further analysis revealed the 2-dimensional PCA configuration to be a poor representation of the higherdimensional structure, by contrast with MDS, as seen when groupings from cluster analyses were superimposed on the 2-dimensional ordinations.

(2) For nematodes, there were no significant differences in species diversity between treatments, but for copepods there were significant global differences, which principally resulted from the contrast between the $H$ replicates and the others. Diversity profiles determined from $k$-dominance curves showed no clear cut treatment effect for nematodes, but a strong effect for copepods, the curves forming a decreasing sequence from high to low diversity in the $\mathrm{C}-\mathrm{L}-\mathrm{M}-\mathrm{H}$ basins (in this case the chances of this are 1 in 24, under a hypothesis of no treatment effect, because there is a predictive direction). For nematodes, total abundance in the $H$ replicates was significantly higher than in $M$ and $C_{\text {, }}$ and copepod abundance was higher in $H$ than in all other treatment levels.

(3) The decrease in diversity of the copepod component of the meiofauna with increasing treatment level was due to a disproportionate increase in abundance of certain species (notably Tisbe spp, but also other species) rather than to selective mortalities. A possible mechanistic explanation for the pollution effects lies in a general response to organic enrichment brought about by the addition of hydrocarbons and/or by mortalities of macrobenthic species; certain opportunistic species, notably Tisbe spp., having a higher colonising potential than others. The toxicity of the pollutants was not an important element in the response, but may have had an effect if the experiment had run for longer or if the pollutants had penetrated into the sediments. The situation in the high dose treatment may therefore represent an early successional stage in the pollution response and did not parallel the condition at the polluted Site $F$ in Frierfjord, where overall densities were much lower than at other sites, and none of the species known to have opportunistic characteristics were found. Therefore, as with the macrofauna, this experiment was not able to determine whether measured levels of pollutants in the field were capable of causing the observed community responses.

\section{Mesocosm microbes}

(1) No multivariate analyses were performed on the microbial data.

(2) Microbes were sampled half-way through the experiment as well as at its termination. Analysis of variance of the various microbial groups indicated that only benthic diatoms showed any significant differences in abundance between treatments. There was a significant increase in diatom abundance in all treatments during the course of the experiment, but pairwise differences between treatments were not consistent on the 2 sampling occasions, nor did they reflect the gradient of dosing levels. There were no differences in biomass of any microbial groups between treatments at the termination of the experiment.

(3) Lack of penetration of pollutants into the mesocosm sediments, or grazing by the increased meiofaunal levels in the high dose boxes, are possible explanations for the lack of response in microbial abundance or biomass with treatment level. 


\section{Mesocosm biomass spectra}

(1) MDS based on geometric size classes within the macrofaunal size range separated the $\mathrm{H}$ replicates from the remainder, but ANOSIM showed that there were only significant differences between $H$ and $M$, and $H$ and $\mathrm{C}$, and then only at the $10 \%$ level. As with the equivalent taxonomic analysis, these results were therefore equivocal.

(2) There were no significant differences in the structure of the overall size spectrum which could be attributed to dosing level.

\section{CONCLUSIONS}

It is appropriate here to consider the relative merits of the different size-categories of organisms which were examined for the GEEP Workshop community studies, both in terms of the practicalities of sampling and processing, and in terms of their resolving power when the same analytical techniques are applied to them.

Macrofauna and meiofauna can be compared on the same terms since sampling regimes and species concepts are similar. Macrofaunal community reponses to pollution have been much more widely studied and a variety of univariate stress indices has been developed, many of which are probably not applicable to the meiofauna (especially those related to size distributions such as B/A and ABC curves, and the use of 'indicator' taxa). Their relative longevity means that the community structure reflects environmental conditions integrated over a long period (years rather than months). Taxonomic literature enabling identification to the species level is also available for many regions of the world, the necessary expertise to do this is present in many laboratories, and methodologies for sampling and processing are well developed. None of these conditions pertain to the meiofauna, but they too have certain advantages. Sampling is less labour intensive in that sample size is smaller, so that sieving need not be done at sea. The taxonomic problems are undoubtedly greater if identification to species level is required, but this workshop has demonstrated that lower levels of taxonomic discrimination produce results which for many purposes are as good as species analyses, for both the meiofauna and macrofauna. Also, the shorter generation times of meiofauna have certain advantages in terms of their faster potential response time to pollution incidents. Meiofaunal studies are hampered by the lack of suitable univariate measures of stress which are applicable to them, and more work needs to be done to develop such techniques. Meiofauna have advantages over macrofauna in experiments to determine cause and effect relationships: because of their size and turnover time, community responses are measurable on the spatial and temporal scales which can be reproduced in such experiments. Also, because of direct benthic development, there are no recruitment problems which are apparent in mesocosm experiments on macrofauna with planktonic larvae. At the species level, copepods were better than the macrofauna or any other meiofaunal taxon at discriminating between sites in the field, and were by far the most sensitive component of the fauna in the mesocosm experiment.

Techniques using microbial communities and size spectra are much less well developed, but this workshop has shown their potential to give qualitatively similar results to the taxonomic analyses of the metazoa. The need to identify microbes only into broad and easily recognisable categories, and the completely ataxonomic size-spectrum analysis, have obvious labour-saving advantages, and here again much more work needs to be directed towards the development of appropriate techniques.

In the determination of pollution effects, a criticism frequently levelled at benthic community studies, in comparison with those of individual organisms, is that the former are highly labour-intensive. In macrofauna studies of the traditional kind, usually many hours have been spent in trying to separate certain difficult groups, such as small spionid, cirratilid or capitellid polychaetes, into species. Analysis of workshop data suggests that very little, if any, information is lost by working at the level of families, which are readily recognisable by ecologists with moderate experience. Useful information is still present when working at the phylum level, and this may also have some conceptual advantages in certain situations. More case studies need to be undertaken to establish the validity of these more cost-effective approaches. 